\title{
New image for the drug industry
}

In this feature by Deborah Cohen (BMJ 2011;343:d7713, doi:10. 1136/bmj.d7713) we said that "the UK spends only $0.9 \%$ of gross domestic product on drugs compared with $2 \%$ in the rest ๑ BMJ Publishing Group Ltd 2011 of the EU," whereas in fact, the European Union spends only $1.2 \%$ on drugs. 